\title{
38
}

\section{Magnetic Field Decay in the Non-superfluid Regions of Neutron Star Cores}

\author{
A.G. MUSLIMOV AND H.M. VAN HORN \\ Department of Physics and Astronomy and C.E. Kenneth Mees Observatory, \\ University of Rochester, Rochester NY 14627-0011 USA
}

\begin{abstract}
We consider a simple model for the evolution of a poloidal magnetic field initally trapped in a region containing normal npe matter within the outer liquid core of a neutron star. We have performed numerical computations for neutron stars with masses of $1.4,1.6$, and $1.7 M_{\odot}$ that undergo very rapid cooling due to the direct Urca process. Because the timescale for the magnetic field decay is directly proportional to $T^{2}$, such a cooling history produces a rapid decline in the magnetic-field strength $B$, even for $B$ as low as $\sim 10^{12} \mathrm{G}$. In particular, we show that an initially quasi-homogeneous magnetic field of strength $B=10^{12} \mathrm{G}$ declines during the first $\sim 1 \mathrm{Myr}$.
\end{abstract}

\subsection{Introduction}

The calculations of Baym, Pethick, and Pines (1969a) have shown that the electrical conductivity of matter in the core of a neutron star is too large to permit ohmic decay of the magnetic field within the age of the Universe. Recently, Haensel, Urpin, and Yakovlev (1990; hereafter HUY) have pointed out that the magnetic-field strength $|\mathbf{B}| \sim 10^{12}$ G typical of pulsars is sufficiently strong that the anisotropy of the transport coefficients cannot be neglected and that the "resistivity" for current flow perpendicular to $\mathbf{B}$ is many orders of magnitude larger than that for current flow parallel to B. Using a simple "toy" model, they found that internal fields $B \geq 10^{13}$ $\mathrm{G}$ can decline to $\sim 10^{12} \mathrm{G}$ in times $\sim 10^{7}$ years, but that fields $\leq 10^{12} \mathrm{G}$ remain practically unchanged on this timescale.

In this paper, we derive an equation for the evolution of the dipole magnetic field, taking into account the anisotropy and nonlinearity of the conductivity tensor produced by the strong field. We solve this equation unmerically for the case in which the field is entirely confined to the onter, 
non-superfluid, normal-npc-matter core of the neutron star. We regard these calculations as a step toward the construction of more complex models of field evolution in neutron stars. We have used the new neutron-star cooling histories obtained by Page and Applegate (1992; hereafter PA) which allow the possibility of the direct Urca process in the neutron star cores. For our present calculations, we have chosen their $1.4,1.6$, and $1.7 M_{\odot}$ models with maximal cooling.

\subsection{Basic Equations and Numerical Results}

In this section, we sketch briefly the derivation of an equation describing the evolution of the magnetic field confined to that part of the core of a neutron star containing normal (i.e., non-superfluid) npe matter. Explicit expressions for the components of the resistivity tensor $\hat{\mathcal{R}}$ under conditions appropriate to neutron star interiors with normal npe matter are presented in Haensel, Urpin, and Yakovlev (1990). For strong fields, they find $\mathcal{R}_{\perp}=\mathcal{R}_{\|}+\Lambda B^{2}$, where $\mathcal{R}_{\perp}$ and $\mathcal{R}_{\|}$are the components of $\hat{\mathcal{R}}$ associated with current flow perpendicular and parallel to $B$, respectively, and $\Lambda$ is a coefficient given below. For this case, an appropriately generalized form of Ohm's Law can be rewritten as

$$
\mathbf{E}=\mathcal{R}_{\| \mathbf{j}}+\mathcal{R}_{H} \mathbf{j} \times \mathbf{B}+\Lambda \mathbf{B} \times(\mathbf{j} \times \mathbf{B}),
$$

where the first term is responsible for ohmic dissipation, and the second represents the Hall drift. ¿From the strong-field relations given by HUY, the coefficients $\mathcal{R}_{\|}$and $\Lambda$ are

$$
\mathcal{R}_{\|}=\frac{m_{\varepsilon}^{*}}{e^{2} n_{\epsilon}} \frac{1}{\tau}{ }_{\epsilon p}, \quad \Lambda=\frac{1}{c^{2} n_{\varepsilon} m_{p}^{*}} \tau_{p n}
$$

where $m_{\varepsilon}^{*} \approx \hbar k_{F}(\epsilon) / c$ is the effective mass of the electron, including relativistic effects, $m_{p}^{*}$ is the effective mass of the proton $\left(\approx 0.8 m_{p}\right.$, cf. Friman and Maxwell 1979), $n_{\epsilon}$ is the number density of electrons, $\tau_{\epsilon p}$ is the electron relaxation time (due to Coulomb scattering from protons), and $\tau_{p n}$ is the proton relaxation time due to scattering from neutrons. According to Baym, Pethick, and Pines $(1969 \mathrm{a}, \mathrm{b})$, the relaxation times $\tau_{\mathrm{ep}}$, and $\tau_{\mathrm{pn}}$ are, respectively,

$$
\tau_{\varepsilon p} \approx 10^{-16} T_{9}^{-2}\left(\frac{\rho}{\rho_{0}}\right)^{5 / 3}\left(\frac{m_{p}^{*}}{m_{p}}\right)^{1 / 2} \mathrm{~s} \text { and } \tau_{p^{n}} \approx 10^{-19} T_{9}^{-2} \mathrm{~s} .(3)
$$

Here $T_{9}=T / 10^{9} \mathrm{~K} ; \rho_{0}=2.8 \times 10^{14} \mathrm{~g} \cdot \mathrm{cm}^{-3}$ is the nuclear-matter density; and other symbols have their usual meanings. 
We now consider the evolution of the dipole component of a purely poloidal magnetic field confined to the neutron star core. Note that the Hall effect disappears in this case. For a purely poloidal field, we can express $\mathrm{B}$ in the form [in spherical coordinates $(r, \theta, \phi)$ ]:

$$
\mathbf{B}=B_{0}\left(2 \frac{S}{r^{2}} \cos \theta \mathbf{e}_{r}-\frac{1}{r} \frac{\partial S}{\partial r} \sin \theta \mathbf{e}_{\theta}\right),
$$

where $B_{0}$ is some normalization magnitude of the field, and $\mathbf{e}_{r}$ and $\mathbf{e}_{\theta}$ are unit vectors in the radial and transverse directions. To obtain the partial differential equation satisfied by the stream function $S$, we first note that the form assumed for $B$ yields a purely azimuthal (and solenoidal) current density $\mathbf{j}$, from Ampère's Law. With the Hall term neglected, eq. (1) yields $\mathbf{E}$ from $\mathbf{B}$ and $\mathbf{j}$. For this case, the $\boldsymbol{r}$ - and $\boldsymbol{\theta}$-components of Faraday's Law can be shown to contain the same physical information. Using Faraday's Law we arrive at the following nonlinear equation for evolution of the function $S(r, t)$ :

$$
\frac{\partial S}{\partial t}=\frac{c^{2} \mathcal{R}_{\|}}{4 \pi}\left\{1+\frac{4}{5} \frac{\Lambda}{\mathcal{R}_{\|}} \frac{B_{0}^{2}}{r^{2}}\left[\left(\frac{S}{r}\right)^{2}+\left(\frac{\partial S}{\partial r}\right)^{2}\right]\right\}\left(\frac{\partial^{2} S}{\partial r^{2}}-2 \frac{S}{r^{2}}\right) .
$$

The main problem with eq. (5) is that even if the field were initially dipolar, higher-order multipoles may be generated due to the third term in expression (1), which we have not taken into account. While the presence of higher-order multipoles can, in principle, modify the evolution of the dipole magnetic field, this problem is beyond the scope of the present paper.

To solve equation (5) we need to specify appropriate boundary conditions. Here, we consider a simplified model in which the internal magnetic field is entirely confined to the core liquid region with normal npe matter (i.e., without superfluidity or superconductivity). For the $1.4 M_{\odot}$ neutron star model we have employed in these calculations (Page and Applegate 1992), the stellar radius is $R_{*}=11.3 \mathrm{~km}$, the radius of the core is $\sim 10 \mathrm{~km}$, with central density $\rho_{c}=4.76 \rho_{0}$, and the normal npe-matter fluid occupies the outer layer of the core, with thickness $\sim 4 \mathrm{~km}$. We take the magnetic field to be confined entirely to the layer of normal npe matter. We assume that both the function $S(r, t)$ and its derivative $\partial S / \partial r$ vanish at the outer and inner boundaries of that region. We also adopt the normalization value $B_{0}=$ $0.5 \times 10^{12} \mathrm{G}$ (see eq. [4]) corresponding to an initial, quasi-homogeneous magnetic field with field strength $10^{12} \mathrm{G}$ at the magnetic poles.

For the $1.4 M_{\odot}$ model, the internal temperature remains relatively constant, at $T \sim 10^{8} \mathrm{~K}$, during the first $\sim 10^{2} \mathrm{yr}$; then it suddenly drops to $\sim 3 \times 10^{7} \mathrm{~K}$. Thereafter, the temperature decreases monotonically until it 

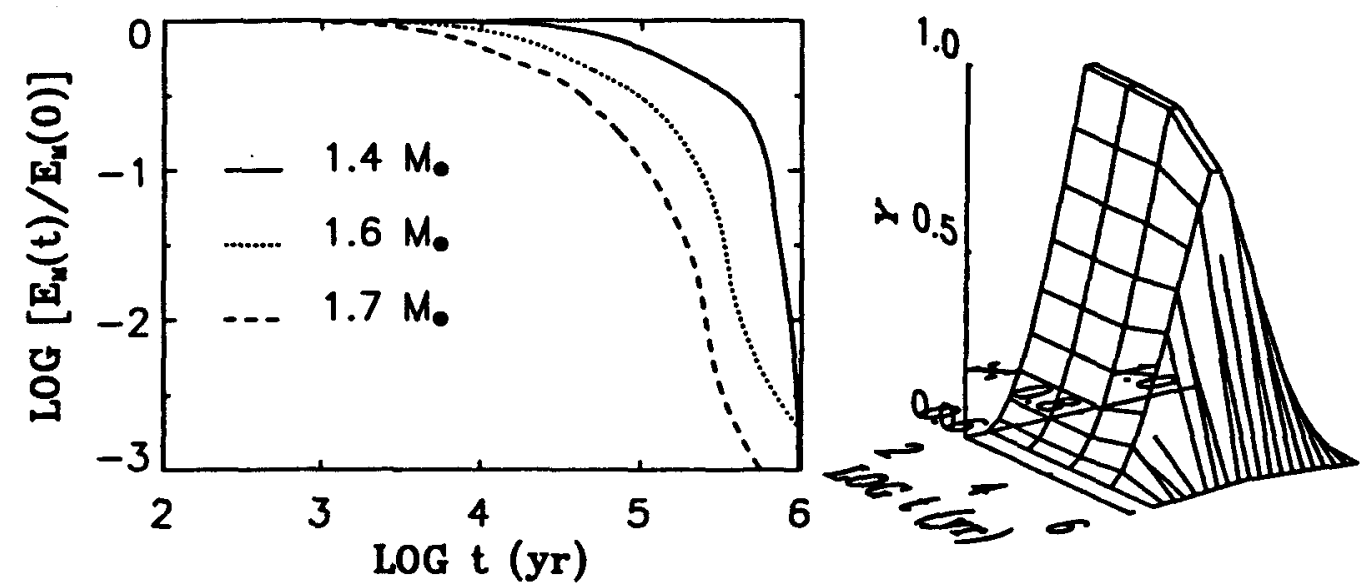

Fig. 30.1 Left panel: The temporal evolution of the magnetic energy, $E_{M}(t)$, normalized to its initial value, $E_{M}(0)$. The magnetic energy decays by a factor of 10 in the first $\sim 4 \times 10^{5}$ years and then drops rapidly toward zero. Curves for the 1.4, 1.6, and $1.7 M_{\odot}$ models of Page and Applegate $(1992$, Fig. 1) are shown. For each case, the initial field strength is normalized by the parameter-value $B_{0}=0.5 \times 10^{12} \mathrm{G}$.

Right panel: The spatial and temporal evolution of the dimensionless Stokes stream function $Y(x, t)=S(x, t) / r_{c}^{2}$, where $x=r / r_{c}$ is the radial distance in units of the core radius $r_{c}$. The calculations are performed for the $1.4 M_{\mathrm{G}}$ neutron star model, and the initial field strength is normalized by the parameter-value $B_{0}=0.5 \times 10^{12} \mathrm{G}$. The stream function is confined to the outer (non-superfluid) core of npe matter, and at the initial time shown, $t=10^{2}$ years, it peaks near $x \approx 0.80$. The shape of $Y$ as a function of $x$ changes very little as $t$ increases, but the amplitude decreases with $t$, at first only gradually, but then more abruptly after $t \geq 3 \times 10^{5}$ years. This is seen more clearly in the left panel.

reaches $\sim 3 \times 10^{6} \mathrm{~K}$ at $t \sim 3 \times 10^{5} \mathrm{yr}$. After $t \sim 3 \times 10^{5}$ yr the internal temperature drops catastrophically. The more massive models have similar cooling histories, but the temperature plateaus are somewhat lower, and the temperature drops happen somewhat earlier than for the $1.4 M_{(:)}$Inodel (see PA).

In the left panel of Fig. 1 we depict the temporal behavior of the ratio $E_{M}(t) / E_{M}(0)$, where

$$
E_{M}(t)=\frac{1}{8 \pi} \int_{V_{B}} B^{2}(\mathbf{r}, t) d^{3} r
$$

is the magnetic energy of the normal fluid layer, with volume $V_{B}$.

The rate of field decay increases as the neutron star cools, and the field effectively disappears at $t \approx 1 \mathrm{Myr}$. The reason for this can be seen by dimensional analysis of eq. (5): with $B_{12}=1 ; \rho=\rho_{0} ; \beta=30 ; T_{9}=$ $3 \times 10^{-3}$, corresponding to the temperature $T=3 \times 10^{6} \mathrm{~K}$ at the time 
$t \sim 3 \times 10^{5}$ yr when the internal temperature begins to drop rapidly; and $R_{6}=0.4$, corresponding to the thickness of the layer of normal npe matter, the characteristic decay timescale can be shown to be $\tau_{\text {decay }}=0.7 \times 10^{6}$ years. (Note that HUY use a definition of $\tau_{\text {decay }}$ that is smaller by a factor of $\pi^{2}$ ). This is comparable to the timescale on which our explicit numerical calculations show that the magnetic energy in the layer with normal npe matter actually does decline. As the left panel of Fig. 1 shows, $E_{M}(t)$ begins to drop rapidly at just the time when the core temperature starts to fall. The temporal evolution of the dimensionless Stokes stream function $Y=S / r_{c}^{2}$, where $r_{c}$ is the radius of the neutron star core, is shown in the right panel of Fig. 1.

Even if there is no proton superconductivity, the presence of superfluid neutrons decreases the value of the parameter $\Lambda$ (see Yakovlev 1993) and makes diffusion of the magnetic field inefficient. If a region occupied by a poloidal magnetic field is surrounded by a superfluid, then the field will be trapped in that region for a time of the order of the Hubble time, provided that the field lines penetrate the superfluid.

\subsection{Conclusions}

Our principal results are that (1) we have proposed a simplified but realistic model equation for the evolution of a dipolar component of the magnetic field in the outer, normal npe-matter core of a neutron star; and (2) we find that the neutron-star cooling, allowing for the direct Urca process, can result in decay of the dipole magnetic field in a normal npe-matter core of a neutron star on a timescale $\sim 10^{6}$ years, even if the initial field is $\leq 10^{12} \mathrm{G}$ Acknowledgements

This work has been supported by NSF grant AST 91-15132 through the University of Rochester, which we gratefully acknowledge. We are also grateful to D. Page for providing us with the numerical data from his neutron star cooling calculations, on which our present calculations are based.

\section{References}

Baym G., Pethick C. and Pines D., Nature, 224, 674, (1969)

Baym G., Pethick C. and Pines D., Nuture, 224, 675, (1969)

Friman B.L., and Maxwell O.V., Ap. J., 232, 541, (1979)

Haensel P., Urpin V.A., and Yakovlev D.G., Astron. Ap., 229, 133, (1990): HUY

Lifshitz E.M., Pitaevskii L.P., Physical Kinetics (Oxford: Pergarnon Press), (1981) Page D., Applegate J.H. Ap. J., 394, L17, (1992): PA

Yakovlev D.G., in Strongly Coupled Plasma Physics, ed. H.M. Van Horn and S. Ichimaru (Rochester, NY: University of Rochester Press), p. 157, (1993) 